\section{AB0808 COMBINATION OF ORAL PARACETAMOL AND TOPICAL NSAIDS FOR OSTEOARTHRITIS PAIN: A SYSTEMATIC SCOPING REVIEW OF THE LITERATURE}

John Bell ${ }^{1}$, Vidhu Sethi ${ }^{2}$, Kamran Siddiqui ${ }^{2}$, Philip G. Conaghan ${ }^{3} .{ }^{1}$ Graduate School of Health, University of Technology Sydney, Sydney, Australia; ${ }^{2}$ GlaxoSmithKline Consumer Healthcare, Singapore, Singapore; ' ${ }^{3}$ Leeds Institute of Rheumatic and Musculoskeletal Medicine, University of Leeds, Leeds, United Kingdom

Background: Topical NSAIDs and paracetamol (APAP) are frequently used for osteoarthritis (OA) pain. ${ }^{1,2}$ In real-world settings, combination treatment is common, ${ }^{3,4}$ with more than one-quarter of patients using topical NSAIDs with oral non-opioid analgesics such as APAP. ${ }^{4}$

Objectives: We conducted a systematic scoping review to: understand current recommendations on concomitant use of topical NSAIDs and APAP for OA pain; evaluate the extent of supporting evidence for this practice; and identify literature gaps.

Methods: We searched PubMed and the Cochrane Library for clinical studies (database inception to January 2019) of topical NSAIDs in OA in which APAP was given concomitantly, either regularly (combination treatment) or as needed (rescue treatment). Grey literature searches were conducted to identify clinical practice guidelines for management of $\mathrm{OA}$ from leading organizations.

Results: After removing duplicates, the literature searches returned 375 articles, of which 66 were clinical studies of topical NSAIDs in OA and were reviewed further. Of these, we identified 1 randomized controlled trial (RCT) evaluating efficacy and safety of combination treatment with APAP and a topical NSAID, in which 43 patients with knee OA currently treated with APAP were randomly assigned to double-blind treatment with ketoprofen plaster or placebo for 4 weeks. ${ }^{5}$ Results showed that the combination of APAP/topical ketoprofen was statistically significantly more effective in pain reduction $(P=0.03)$ and physician's global assessments $(P=0.01)$ compared with APAP/placebo. Of the remaining 65 topical NSAID studies, another $37(57 \%)$ allowed rescue treatment with APAP during the treatment period (including 34 RCTs; 1 open-label extension of 2 RCTs; 1 patient preference study; and 1 single-arm open clinical trial). Because none of these studies randomly assigned patients to the combination, it was not possible to evaluate efficacy or safety of the combination based on these data. Review of OA guidelines identified 3 major organizations-EULAR, NICE, and ESCEO-that recommend or allow concomitant use of topical NSAIDs and APAP for specific joints. ${ }^{6-8}$

Conclusion: In clinical practice, drug combinations are frequently used to manage OA pain, and some OA treatment guidelines allow the use of topical NSAIDs concomitantly with APAP. Very limited supporting data are available, with only a single small RCT demonstrating the efficacy and safety of the combination. However, it may be inferred from the fact that more than half of the topical NSAID studies identified allowed APAP rescue therapy that this combination is generally perceived to be efficacious and well tolerated. Additional, larger RCTs are needed to confirm the benefit of this commonly used analgesic combination in managing $\mathrm{OA}$ pain

\section{REFERENCES}

[1] Conaghan PG, et al. Clin Rheumatol. 2015;34:1581-8.

[2] Jackson H, et al. BMJ Open. 2017;7:e019694.

[3] Gupta R, et al. Int J Res Med Sci. 2018;6:985-9.

[4] Wilson N, et al. Rheumatology. 2015;54:860-7.

[5] Yoo B, et al. J Korean Rheum Assoc. 1996;3:70-5

[6] Bruyere O, et al. Semin Arthritis Rheum. 2014;44:253-63.

[7] Geenen R, et al. Ann Rheum Dis. 2018;77:797-807.

[8] NICE. Guideline CG177. 2014.

Disclosure of Interests: John Bell Consultant for: John Bell has acted as consultant for Bayer, GlaxoSmithKline, Mylan, Novartis, Pfizer and Reckitt Benckiser., Speakers bureau: John Bell has received payment for speaking from GlaxoSmithKline, Pfizer and Reckitt Benckiser., Vidhu Sethi Employee of: Vidhu Sethi is an employee of GlaxoSmithKline Consumer Healthcare, Singapore., Kamran Siddiqui Employee of: Kamran Siddiqui is an employee of GlaxoSmithKline Consumer Healthcare, Singapore., Philip G Conaghan Consultant for: Flexion Therapeutics, AbbVie, Medivir, Merck Serono, Novartis, GlaxoSmithKline
DOI: 10.1136/annrheumdis-2019-eular.7257

\section{AB0809 A STUDY TO COMPARE ARTICULAR CARTILAGE VOLUME IN HEALTHY AND OSTEOARTHRITIC KNEE AND ITS CORRELATION WITH CLINICO - RADIOLOGICAL SEVERITY}

Sudeepti Srivastava, Rajeswar Nath Srivastava, Amar Chandra Sharma. King George's Medical University, Orthopaedic Surgery, Lucknow, India

Background: Knee Osteoarthritis (KOA) is a persistent debilitating disease characterized by loss of articular cartilage. Known to be a disease of elderly, it may not be necessarily so, as the loss in articular cartilage volume (ACV) begins early but detected very late on X-rays and by that time no therapy works and joint replacement is the only answer.

Objectives: To determine the role of ACV in early diagnosis of KOA, which may not only monitor disease progression from a very early stage it may also open a gateway to slow down or stop the structural changes involved in KOA.

Methods: 60 Cases and equal number of age, sex matched controls were recruited. Each subject had MRI of the reference knee. ACV was measured manually by means of image processing on an independent workstation using semi-automated machine GE Signa. VAS for knee pain and WOMAC for pain, stiffness and disability were recorded for clinical severity and $\mathrm{X}$-rays for radiological severity by $\mathrm{KL}$ grading.

Results: With age, a statistically significant inverse correlation of ACV was found in both healthy and osteoarthritis knees. Height, weight and BMI were independent of ACV in healthy knee. In KOA, a significant positive correlation of ACV was observed with height, a significant inverse correlation with WOMAC scores and significant difference with sub- scales of WOMAC index (pain, stiffness and physical function). The statistical difference in ACV between the two categories of WOMAC scores $(<32$ and $>32$ ) was significant.

Conclusion: This study concluded that ACV is significantly lower in osteoarthritis knee as compared to healthy adult knee. This study also found that KOA in females occurs at an early age and progresses slowly whereas in males occurs late and progresses rapidly. ACV may become one of the most promising tools for early diagnosis of KOA and in monitoring disease progression.

\section{REFERENCE}

[1] Beary JF III. Joint structure modification in osteoarthritis: development of SMOAD drugs. CurrRheumatol Rep 2001;3:506-512.

Disclosure of Interests: None declared

DOI: 10.1136/annrheumdis-2019-eular.6708

\section{AB0810 INTRA-ARTICULAR CNTX-4975 FOR PAINFUL KNEE OSTEOARTHRITIS: ASSESSMENT OF COOLING METHODS FOR REDUCING PROCEDURAL PAIN}

Randall Stevens $^{1}{ }^{1}$ Kimberly Guedes ${ }^{1}$, Nilam Mistry ${ }^{1}$, Duncan Lascelles ${ }^{1}$, David Ball ${ }^{2} .{ }^{1}$ Centrexion Therapeutics Corp, Boston, United States of America; ${ }^{2}$ MAC Clinical Research, Manchester, United Kingdom

Background: CNTX-4975 is a long-acting, trans-capsaicin injection in phase 3 trials for treatment of moderate to severe pain associated with knee osteoarthritis (OA). Intra-articular (IA) CNTX-4975 injection produces short-lived procedural pain that can be ameliorated with joint cooling. A prior analysis (Cohort 2) demonstrated that a circumferential circulating ice water wrap (CCIWW) more effectively lowered IA knee temperature and reduced procedural pain than an ice pack on top of the knee (3.4 vs 7.3, respectively, on a numeric pain rating scale [NPRS] 10 minutes post-CNTX-4975).

Objectives: Subjects were enrolled in 2 separate cohorts (C3/C4) to: compare effects of 2 circumferential cooling methods on IA and skin knee temperature and procedural pain (C3); and assess procedural pain with an abbreviated cooling schedule and with vs without post-CNTX4975 cooling (C4)

Methods: Eligible subjects were adults aged $45-75$ yrs with $\geq 3$ months of bilateral moderate to severe $O A$ knee pain. All subjects received each 